\title{
Comentario a propósito del Protocolo de Actuación Policial de la Secretaría de Seguridad Pública del Distrito Federal (México), para la Protección, Rescate, Concientización, Respeto y Bienestar Animal ${ }^{1}$
}

\author{
Eder Ramirez Galindo ${ }^{2}$
}

\section{INTRODUCCIÓN}

El 3 de marzo del presente año, el Dr. Jesús Rodríguez Almeida, Secretario de Seguridad Pública del Distrito Federal, remitió el acuerdo 06/2014 por el que se expide el Protocolo de Actuación Policial de la Secretaria De Seguridad Pública del Distrito Federal, para la Protección, Rescate, Concientización, Respeto y Bienestar Animal. Protocolo que tiene como principios de interpretación y aplicación el respeto a la vida animal, el bienestar animal, la legalidad, racionalidad, congruencia, oportunidad y proporcionalidad. Este protocolo viene a ser un parteaguas sobre cómo deben actuar las autoridades policiales cuando se encuentre en riesgo el bienestar de un animal, y sobre la necesidad de un grupo especializado en tratándose de situaciones que involucren la afectación o detrimento en la integridad de un animal.

El Distrito Federal ha sido la entidad en el territorio mexicano pionera en torno a la protección de los animales; muestra de ello es el acuerdo por el que se crea el Protocolo de Actuación Policial que ahora se analiza. Por ser el primero en su especie este protocolo merece especial consideración, ya que con su creación se distingue que debe existir una forma especial en que las autoridades se deban conducir cuando se les presente una contingencia en relación con un menoscabo en el bienestar de los animales. El presente comentario tratará de explicitar lo contenido en el protocolo materia de análisis, vertiendo consideraciones al respecto.

\section{SUMARIO}

\footnotetext{
${ }^{1}$ http://www.derechoanimal.info/bbdd/Documentos/1503.pdf

2 Abogado. Licenciado en Derecho por la Universidad Michoacana de San Nicolás de Hidalgo. Miembro del Grupo de Investigación en Derecho Animal (GIDA) de la Facultad de Derecho y Ciencias Sociales de la Universidad Michoacana de San Nicolás de Hidalgo. Estudiante de la Maestría en Derecho Procesal Constitucional, UMSNH (Morelia, Michoacán, México).
} 
1. La Brigada de Vigilancia Animal

2. Objeto del Protocolo de Actuación Policial

3. Reporte o denuncia ciudadana

4. Atención de denuncias

5. Protocolo de actuación

6. Supuestos de atención

7. Canalización de la fauna

8. Vinculación, información y difusión

9. Conclusiones

\section{LA BRIGADA DE VIGILANCIA ANIMAL}

Cabe destacar que el protocolo en comento va dirigido principalmente a la denominada Brigada de Vigilancia Animal, un grupo policial integrante de la Secretaria de Seguridad Pública del Distrito Federal ${ }^{3}$. Grupo policial que deberá ser actualizado y especializado en torno al marco normativo nacional e internacional en materia de protección y bienestar animal, el comportamiento animal, contención de animales domésticos y silvestres, la tenencia responsable de los animales, importancia de las zoonosis, bioseguridad, rapel, primeros auxilios, riesgos laborales y el bienestar animal, atendiendo a las cinco libertades fundamentales de los animales, a saber, libre de sed y hambre, libre de incomodidad, libre de miedo y angustia, libre de dolor lesiones y enfermedad, libre para comportarse de manera normal, cuidando adicionalmente la integridad física de las personas y bienes.

\section{OBJETO DEL PROTOCOLO DE ACTUACIÓN POLICIAL}

El Protocolo de Actuación Policial tiene por objeto atender los reportes y denuncias de maltrato, negligencia en el cuidado y rescate de animales, domésticos o silvestres, que se encuentren en propiedad privada o pública, o en el interior de vehículos.

\section{REPORTE O DENUNCIA CIUDADANA}

Cualquier ciudadano está legitimado para denunciar cualquier hecho relativo al maltrato de animales a la Brigada de Vigilancia Animal, ya sea por vía telefónica, por

\footnotetext{
${ }^{3}$ http://www.ssp.df.gob.mx/PartCiudadana/Pages/Brigada-de-Vigilancia-Animal.aspx
} 
escrito, por medios de comunicación electrónica o mediante solicitud de apoyo interinstitucional.

Para el caso de denuncia telefónica, el centro de atención del secretario, en coordinación con el call center de la Secretaria de Seguridad Pública y la aplicación para teléfonos móviles "Mi Policía k8", serán los que reciban las denuncias ciudadanas.

En lo que se refiere a la denuncia por escrito, ésta debe presentarse ante la oficialía de pares de la Subsecretaría de Participación Ciudadana y Prevención del Delito, la Dirección General de Prevención del Delito, la Dirección Ejecutiva de Aplicación de Programas Preventivos Institucionales o el Centro de Atención del Secretario.

Por lo que ve a los medios electrónicos, no se define qué se debe entender por ellos, pero acorde a la página web de la Secretaría de Seguridad Pública del Distrito Federal, se presume que se refiere a correo electrónico o bien mediante redes sociales, por lo que se pueden realizar denuncias por estos medios, las cuales deberán ser atendidas.

$Y$ en cuanto a los apoyos interinstitucionales, debemos entender que la Brigada de Vigilancia Animal trabaja en conjunto con la Procuraduría General de la República, la Procuraduría Federal de Protección al Ambiente, la Procuraduría General de Justicia del Distrito Federal, la Secretaria del Distrito Federal y Órganos Político Administrativos, la Procuraduría Ambiental y de Ordenamiento Territorial del Distrito Federal y el Instituto de Verificación Administrativa del Distrito Federal.

\section{ATENCIÓN DE DENUNCIAS}

La Brigada de Vigilancia Animal atenderá toda denuncia relativa a la comisión de un delito tipificado por el Código Penal o de una infracción a la Ley de Protección Animal y su Reglamento, tales como la crueldad y maltrato animal o pelea de perros, en el primer caso, $o$ animales que se encentren en plantones o manifestaciones o anomalías en centros y establecimientos que se dediquen a la venta de animales, así como de todo aquel acto que signifique un detrimento a los principios que el protocolo comentado señala.

\section{PROTOCOLO DE ACTUACIÓN}

El proceso de actuación empieza con la comunicación de la denuncia ante las autoridades ya mencionadas por los canales previstos. Una vez que la autoridad tiene 
conocimiento de los hechos se procede a comunicarlos a la Brigada de Vigilancia Animal, la cual integrará un grupo de trabajo que se presentará en el lugar de los hechos. Una vez que haya arribado, el grupo de trabajo valorará la situación y verificará si los hechos denunciados son ciertos o no. En caso de que no sean ciertos, dará razón al mando responsable, pero en el caso de que sean ciertos, realizará una inspección ocular, la cual le permitirá actuar acorde a las circunstancias y con base en los supuestos de actuación que el mismo protocolo señala. En caso de existir daño a bienes o personas, lo comunicará a los servicios de emergencia correspondientes. Finalmente, elaborará un informe policial, previo folio solicitado a la base operativa de radio, donde se asentarán las circunstancias de modo, tiempo y lugar de la actuación, así como la descripción de las personas o animales involucradas y cualquier otro aspecto relevante, al grupo de trabajo de la Brigada de Vigilancia Animal.

\section{SUPUESTOS DE ATENCIÓN}

La Brigada de Vigilancia Animal debe atender a las circunstancias particulares que se le presentan apegado a los supuestos de actuación referidos en el protocolo de actuación policial: casos referidos a maltrato o crueldad animal, rescate de animales, agresión animal, venta de animales en vía pública y en vehículos, animales que se encuentren en plantones o manifestaciones, anomalías en centros o establecimientos que se dediquen a la venta de animales, peleas de perros, entrega voluntaria de animales, posesión de animales silvestres, adiestramiento de animales en vía pública y actuaciones en caso de animales lesionados o estresados.

Cabe señalar que, si bien cada supuesto conlleva su peculiaridad, existe un patrón de seguimiento, como lo es:

1. Identificación de la Brigada de Vigilancia Animal con el propietario, poseedor encargado o terceros que se encuentren en relación con el o los animales.

2. Información del motivo de la visita.

3. Solicitar autorización para revisar a los animales.

4. En caso de actos u omisiones que sean constitutivos de delito o falta administrativa, se le informará al propietario, poseedor encargado o tercero que se encuentren en relación con el o los animales, las sanciones de las que pudieran ser acreedores.

5. Si no se permitiera la revisión, se procederá a presentar formal denuncia correspondiente.

6. Cuando se haga entrega voluntaria de los animales, se dejará constancia debidamente requisitada. 
7. Los animales serán trasladados al centro correspondiente de atención animal, para su protección y resguardo.

8. Después de resguardo temporal, se canalizarán a las asociaciones protectoras de animales.

9. Si no se encontrara destino para los animales, se les enviará al centro de atención canina del Distrito Federal dependientes de la Secretaria de Salud.

10. Si no se hiciera entrega voluntaria de los animales, se darán recomendaciones a seguir por parte del propietario, poseedor o terceras personas relacionadas con el animal.

11. Se realizarán visitas de seguimiento.

Mención especial merece que, tratándose de rescate de animales, previo análisis de la situación y de la aplicación de contención de animales, traslado de animales y base médica, se entregarán éstos a su propietario, encargado o responsable, siempre y cuando no se encuentren protegidos como animales silvestres por algún ordenamiento jurídico aplicable, pues en tal caso se procederá a la entrega de ellos al Centro para la Conservación en Investigación de la Vida Silvestre de los Reyes la Paz Estado de México, dependiente de la Secretaría de Medio Ambiente y Recursos Naturales.

En caso de que los actos verificados por la Brigada de Protección Animal sean constitutivos de delito en flagrancia o de falta administrativa, se detendrá a las personas infractoras o probables responsables, llevándolos ante la autoridad competente. Se tratará de situaciones de maltrato o crueldad animal, pelea de perros, posesión ilegal de animales protegidos, venta de animales en vía pública o en vehículos, o adiestramiento de animales en vía pública.

En caso de agresión animal, animales lesionados o estresados, una vez contenido el animal, se procederá a trasladarlo al centro de atención canino, para una revisión sanitaria.

Un último apunte sobre lo anterior, es el relativo a dos supuestos en los que se puede llevar a cabo esta actuación: en primer lugar, las verificaciones hechas en domicilio privado; y en segundo lugar, las realizadas en vía pública. En todos los casos se debe atender a las garantías constitucionales de seguridad jurídica y de debido proceso. Así, en caso de que se llegara a pretender una actuación en domicilio privado, será necesaria la autorización de la persona idónea a tal efecto, pues en caso contrario se tendrá que acudir a levantar formal denuncia y proceder conforme a Derecho proceda. Situación que no acontecerá, sin embargo, en las actuaciones en vía pública, donde se puede actuar de una manera más rápida, por encontrarnos en algún momento sobre la hipótesis de flagrancia. 
Para una mejor comprensión del proceso que se realiza en torno a una atención sobre cualquiera de los supuestos enunciados, se muestra el siguiente esquema:

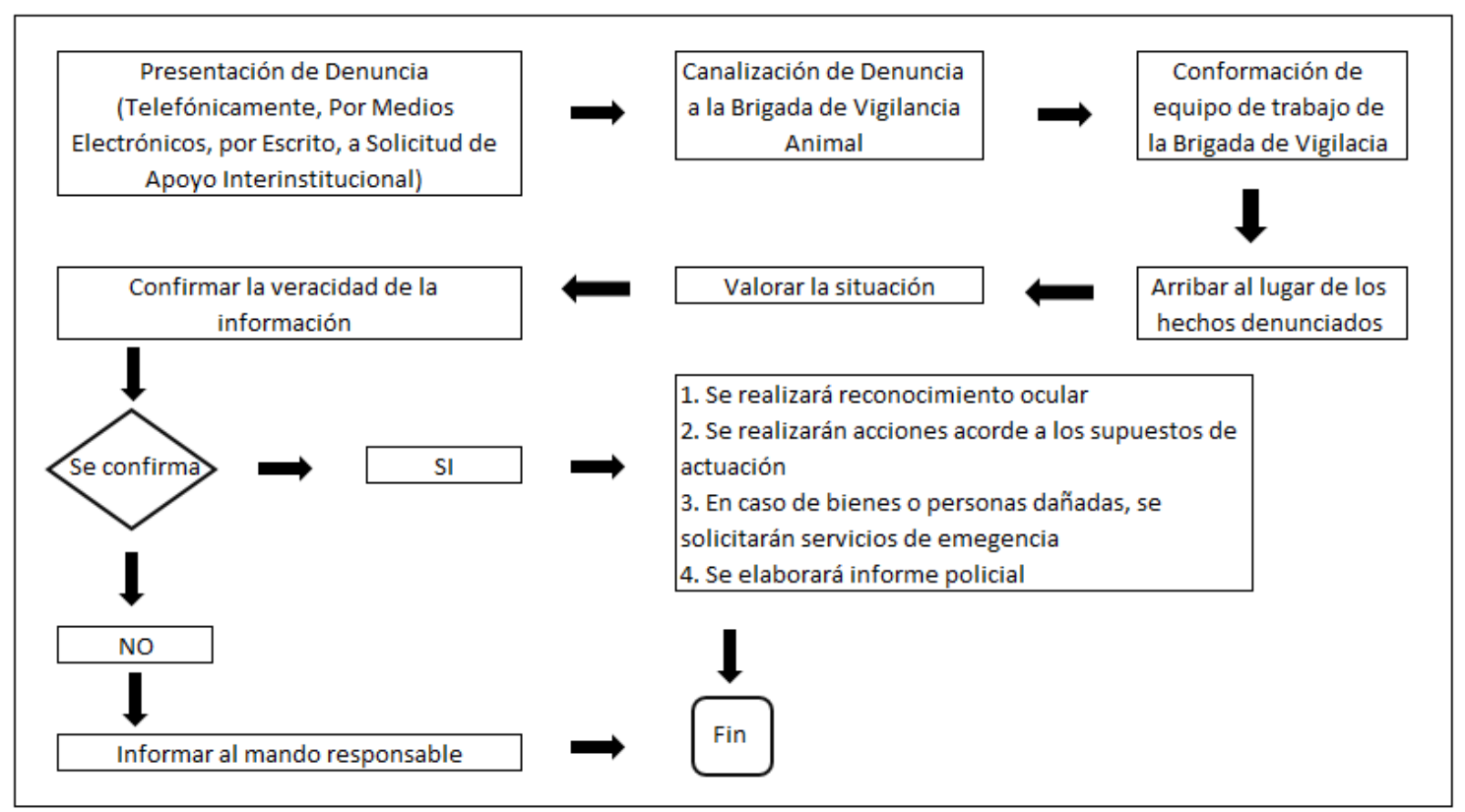

Cuadro 1

\section{CANALIZACIÓN DE FAUNA}

Así como se habló del apoyo interinstitucional para recibir las denuncias y para proceder a la realización del Protocolo, también existe apoyo interinstitucional para la canalización de la fauna, acorde al tipo o especie de que se trate.

Tratándose de animales de fauna silvestre se canalizará al Centro de Conservación e Investigación de Vida Silvestre de la Secretaría del Medio Ambiente y Recursos Naturales. Si se refiere a animales de producción, el canal será la Secretaría de Agricultura, Ganadería, Desarrollo Rural, Pesca y Alimentación. Los Centros de Atención Caninos y Órganos Político-Administrativos a través de las Clínicas Veterinarias Delegacionales, serán los encargados de resguardo, prevención y control de caninos y felinos. Por último, las Asociaciones Protectoras de Animales son las encargadas de recibir caninos y felinos, mediante solicitud formal para su resguardo temporal, auxiliando en el proceso de adopción que garantice el bienestar animal.

\section{Vinculación, información y difusión}


Uno de los aspectos más importantes que realiza la Brigada de Protección Animal es la de dar a conocer información y promover el bienestar animal; para tal efecto, se sirve realizar sesiones informativas y eventos masivos, así como capacitar o realizar pláticas o talleres, con la finalidad de difundir la cultura de protección y bienestar animal. Para todo lo cual tiene vinculación con instituciones o universidades, tanto del sector público o privado.

\section{Conclusiones}

La creación de la Brigada de Vigilancia Animal en el Distrito Federal como entidad del sector de Seguridad Pública ha sido un gran avance en el Estado Mexicano, ya que esto permite tener una especialización en el trato y conducción de animales cuando nos encontramos ante los supuestos de los que hemos venido hablando. Es evidente que dicha especialización resulta necesaria, pues no se puede seguir en estos casos el mismo protocolo de actuación policial aplicable a situaciones que sólo versan sobre personas humanas.

El Protocolo de Actuación Policial y la Brigada de Vigilancia Animal no sólo resultan de importancia para la protección de los animales, sino que constituyen además un gran avance en el universo jurídico, pues se reconoce la trascendencia e importancia que los animales representan, dejando de lado la antigua concepción de equiparar a un animal con una cosa.

El protocolo que ahora se estudia establece que toda persona puede denunciar hechos constitutivos de afectación a los animales; sin embargo, debemos tener claro que dichas "denuncias" sólo se deben considerar como denuncias de hechos, y no denuncias penales, ya que las mismas no integran una averiguación previa, por lo que la Brigada de Protección Animal, en atención al derecho de debido proceso, tiene limitación de actuación cuando se trata de la revisión de un animal en un domicilio particular, por lo que debe presentar formal denuncia a autoridad competente, que en caso de delitos, sería el Ministerio Público.

Este Protocolo de Actuación Policial permite a la Brigada de Vigilancia Animal tener la directriz de manejo ante los supuestos que versen sobre una afectación o menoscabo en el bienestar de un animal. En este sentido, si bien es cierto que sólo se habla de ciertos supuestos de actuación, el mismo protocolo refiere que se trata de una lista de supuestos que se elabora de manera enunciativa, mas no limitativa; por tanto, cualquier afectación de la que pueda ser objeto un animal dentro del territorio del 


\section{dA derecho ANIMAL la web center de los animales con derecho}

Distrito Federal, contemplada en algún ordenamiento relativo a la materia, será objeto de competencia de la brigada multicitada.

Por último, no debe escaparse el hecho de que la Brigada de Protección Animal no sólo actúa en las situaciones emergentes de riesgo para el bienestar de un animal, sino que dentro de las acciones que realiza se encuentran también la educación y difusión de una cultura protectora de los animales, concienciando a la población en general, y evitando con ello que se puedan llevar a cabo actos $u$ omisiones que afecten a los animales, toda vez que es bien sabido que es mejor fomentar una cultura de prevención, que de reparación.

Bellaterra. Barcelona, 16 de diciembre de 2014 